\title{
Los programas de movilidad estudiantil en la Educación Superior: el caso de una universidad española
}

\section{Student mobility programs in higher education: the case of a Spanish university}

\author{
DOI: $10.46932 / \mathrm{sfjdv} 2 \mathrm{n} 2-028$
}

Received in: january 1st, 2020

Accepted in: March 30th, 2020

\author{
Sandra Arroyo Salgueira \\ Investigadora Predoctoral (REF: UAFPU2019B-09) \\ Institución: Universidad de Alicante
}

Dirección: Facultad de Educación. Carretera San Vicente del Raspeig s/n, 03690. San Vicente del Raspeig - Alicante

\section{Dr. Marcos Jesús Iglesias Martínez}

Profesor Titular de Universidad

Dirección: Facultad de Educación. Carretera San Vicente del Raspeig s/n, 03690. San Vicente del

Raspeig - Alicante

E-mail: marcos.iglesias@ua.es

Dra. Inés Lozano Cabezas

Profesora Contratada Doctora

Institución: Universidad de Alicante

Dirección: Facultad de Educación. Carretera San Vicente del Raspeig s/n, 03690. San Vicente del Raspeig - Alicante

E-mail: ines.lozano@ua.es

\section{RESUMEN}

En el proceso de construcción del Espacio Europeo de Educación Superior (EEES) se inicia una reforma de las instituciones universitarias que trae consigo muchos cambios en la Educación Superior. Entre ellos, la adopción de un sistema de titulaciones que favorezca la equiparación de los estudios, la calidad en la enseñanza y la movilidad estudiantil. Con respecto a esta última, la Unión Europea (UE) pone a disposición de las universidades programas de movilidad para toda la Comunidad Educativa. Con el objetivo de conocer la disponibilidad de estos programas en la Universidad de Alicante (UA), nos proponemos recopilar la información disponible y realizar un análisis de sus características que nos permita determinar si se promueven las mismas oportunidades de movilidad para todo el alumnado de los diferentes centros. Los resultados nos muestran que algunos centros ofertan pocos programas de movilidad y no todos disponen de ayudas para prácticas. Además, las cuantías de las ayudas son pequeñas y, aunque la UA intenta compensarlo con aportaciones propias, parte del alumnado no podrá permitirse realizar movilidad durante sus estudios. En general, esto crea un contexto de discriminación del alumnado, por cuestiones de disponibilidad de programas en algunas facultades y por cuestiones económicas, que debe ser resuelto en el seno de la UA y la UE respectivamente.

Palabras clave movilidad estudiantil, Educación Superior y oportunidades educacionales. 


\begin{abstract}
In the process of building the European Higher Education Area (EHEA), a reform of university institutions is being initiated that brings with it many changes in Higher Education. Among them, the adoption of a degree system that favors the equalization of studies, the quality of teaching and student mobility. With regard to the latter, the European Union (EU) provides universities with mobility programs for the entire educational community. In order to know the availability of these programs at the University of Alicante (UA), we propose to collect the available information and perform an analysis of its characteristics that allow us to determine whether the same mobility opportunities are promoted for all students from different centers. The results show that some centers offer few mobility programs and not all of them have grants for internships. In addition, the amounts of the grants are small and, although the UA tries to compensate for this with its own contributions, part of the student body will not be able to afford mobility during their studies. In general, this creates a context of discrimination of students, due to issues of availability of programs in some faculties and economic issues, which must be resolved within the UA and the EU respectively.
\end{abstract}

Keywords: student mobility, Higher Education and educational opportunities.

\title{
1 INTRODUCCIÓN
}

Tras la Declaración de Bolonia (1999), un acuerdo realizado por 29 países que sienta las bases para la construcción del Espacio Europeo de Educación Superior (EEES), se inicia un proceso de reforma de las propias universidades, las administraciones educativas, los equipos de gobierno, las asociaciones y las redes universitarias. En este espacio se promueven mejoras en torno a los principios de calidad, movilidad, diversidad y competitividad universitarios. Esto se concreta en planteamientos que pretenden la promoción y excelencia de la Educación Superior europea; la adopción de un sistema de titulaciones que facilite la movilidad de docentes y alumnado; el respeto a la diversidad educativa y cultural de Europa; y el fomento de la competitividad del sistema universitario europeo en el ámbito internacional (EEES, 2019).

Entre las políticas implementadas para mejorar la calidad de la enseñanza e incentivar la movilidad estudiantil destacamos un sistema de titulaciones basado en créditos, European Credit Transfer System (ECTS), que favorece la comprensión de los planes de estudios realizados en cualquier institución europea y la comparación de los mismos. Asimismo, con unas estructuras y contenidos más próximos para las diversas titulaciones europeas, se promueve el uso de las herramientas Europass (Perfil, certificado y documento de movilidad) gracias a la implantación del Suplemento Europeo al Título (SET) que permite al alumnado solicitar más fácilmente la homologación de los títulos universitarios en otros países (EEES, 2019; MECD, 2019).

Todas estas facilidades son un aliciente para el alumnado universitario que puede ver internacionalizados sus estudios y promovida su empleabilidad gracias a la movilidad académica (Cairns, Krzaklewska, Cuzzocrea, \& Allaste, 2018). Además, traen consigo beneficios para las instituciones 
universitarias que aumentan su prestigio social y establecen redes de contactos internacionales muy provechosas para el intercambio de experiencias con expertos y la generación de proyectos de investigación internacionales (Ramírez, \& Ortega, 2019). Aunque en este camino debemos se conscientes de la necesidad de promover la igualdad de oportunidades en el acceso a todos los servicios universitarios. Esta dimensión social, premisa que favorece acercar las enseñanzas a las necesidades sociales a través de sistemas educativos más sensibles al cambio social (Consejo Europeo, 2016), busca tener en cuenta los privilegios socioeconómicos de los aspirantes y los distintos países miembros a la hora de repartir los recursos y las ayudas a la movilidad europeos (Yuval \& Miri, 2017). Este reparto equitativo permitiría al alumnado universitario desarrollar sus estudios o realizar prácticas en más de una institución (Arroyo y Lozano, 2020), ya que se trata de un valor que posibilita el acceso de toda la comunidad universitaria a los recursos que el EEES pone a su disposición, así como un medio para la no discriminación del alumnado por cuestiones económicas.

Para conocer el estado actual de la cuestión recurrimos a las estadísticas (Eurydice, 2017; Unidad Técnica de Calidad de la Universidad de Alicante, 2019) que nos indican que los niveles de movilidad entre universidades son todavía bajos; en el caso de la UA el total de alumnado con beca en un programa de movilidad son 866 personas (curso 2017-2018) de un total de 21.949 estudiantes de grado (curso 20182019). También que estamos todavía lejos de cumplir con los Indicadores de la Movilidad en la Educación Superior en lo que respecta a información y orientación al alumnado, preparación en lenguas extranjeras y portabilidad de becas y préstamos; a pesar de lo comentado anteriormente y de las herramientas disponibles, también en el reconocimiento de los resultados de aprendizaje y las cualificaciones alcanzadas puesto que el reconocimiento no se aplica en muchas titulaciones; y como ya habíamos visto, en apoyo a los estudiantes procedentes de un entorno socioeconómico desfavorecido (Eurydice, 2017).

Por otro lado, según el informe BET 2011-13 los elementos que afectan la movilidad son los visados, los permisos de trabajo/investigación, la documentación legalizada, etc. Por tanto, es aquí donde los expertos nos remarcan la necesidad de optimizar los trámites administrativos que repercuten en la movilidad del alumnado. En nuestro caso, facilitando los trámites que se recogen en las convocatorias de cada programa y mejorando la normativa que la Universidad de Alicante tiene al respecto ( $\underline{B O U A \text { del } 5}$ de noviembre de 2010 normativa de movilidad de estudiantes de la Universidad de Alicante).

Otros estudios señalan como determinantes de la movilidad: la proximidad, el coste de la movilidad o la disponibilidad de una beca o ayuda familiar para poder realizarla; la presencia de otros estudiantes internacionales; o factores como el clima, la cultura, las actividades deportivas, sociales y de ocio (Sin, Tavares \& Neave, 2017). También, en algunos casos, la percepción de la calidad de la docencia impartida o la seguridad del destino (Donat, Carmona, Vidal, \& Benlloch, 2018). Todos ellos esenciales 
a la hora de seleccionar el destino como si de un viaje turístico se tratara. Así, las universidades son vendidas como una marca que debe estar presente en RRSS y promocionarse como un destino de ocio y diversión para el alumnado extranjero (Nicholls, 2018; Cuzzocrea \& Cairns, 2020). Aunque esto no quita la necesidad de visibilizar las oportunidades de movilidad (Ramos, Sánchez, Saucedo, \& Cortés, 2021) que las universidades ofrecen a su alumnado con portales web que ofrezcan información práctica (Eurydice, 2017; European Commission/EACEA/Eurydice, 2020). Esto permitirá mejorar el conocimiento de los distintos escenarios formativos, investigadores y culturales que se dan en las universidades europeas para que los participantes de los programas de movilidad se sientan más apoyados a la hora de dar el paso y seleccionar la institución de destino.

Asimismo, será necesario contar con un sistema de garantía de la calidad del programa y la institución, que asegure el reconocimiento de los estudios cursados en estas instituciones europeas (Eurydice, 2017); que permita mejorar las estrategias institucionales en la acogida del alumnado, la dotación económica necesaria para llevar a cabo la movilidad en todo el territorio europeo de manera equitativa y la flexibilización de los programas formativos para llevar a cabo con éxito esta práctica (Sin, Tavares \& Neave, 2017).

En nuestro trabajo, nos planteamos analizar la disponibilidad y las características de los programas de movilidad universitarios implementados en la Universidad de Alicante para determinar si, como principal cuestión de investigación, se promueven las mismas oportunidades de movilidad para todo el alumnado de la UA.

\section{MÉTODO}

Para poder analizar la disponibilidad y las características de los distintos programas de movilidad que se ofrecen al alumnado, nos hemos propuesto tres objetivos específicos:

1. Revisar los programas de movilidad que la Universidad de Alicante ofrece a su alumnado.

2. Conocer las características de los programas de movilidad que ofrece la UA.

3. Analizar las diferencias que presentan los distintos centros de la UA con respecto a la posibilidad que tiene su alumnado de acceso a becas de movilidad.

En esta labor nos proponemos recopilar la información disponible, a cerca de los programas de movilidad para el alumnado, en la web de las diferentes facultades y la del Secretariado de Movilidad. 
Luego procederemos a elaborar los documentos que nos permitan comparar la información de cada centro con el resto. Para ello accederemos a las bases de las convocatorias y a la normativa interna donde recopilaremos las características más notorias de cada programa. Finalmente, trataremos de determinar las diferencias que existen en el acceso a estos programas para el alumnado de los diferentes centros.

\section{ANÁLISIS Y PRESENTACIÓN DE LOS RESULTADOS}

\subsection{REVISAR LOS PROGRAMAS DE MOVILIDAD QUE LA UNIVERSIDAD DE ALICANTE} OFRECE A SU ALUMNADO.

A continuación, se muestran los resultados obtenidos en el proceso de búsqueda y documentación de las becas ofertadas en los diversos centros de la Universidad de Alicante. Para ello hemos elaborado las siguientes tablas, la primera contiene un listado de las becas existentes en la Universidad de Alicante y su oferta en cada una de las facultades (véase tabla 1); en la segunda aparecen los requisitos y características de las becas que más se promueven desde la Universidad de Alicante (véase tabla 2).

Tabla 1. Movilidad por centros de la Universidad de Alicante.

\begin{tabular}{|c|c|c|c|c|c|c|c|}
\hline $\begin{array}{l}\text { Becas de } \\
\text { movilidad }\end{array}$ & $\begin{array}{l}\text { Facultad de CC } \\
\text { económicas y } \\
\text { empresariales }\end{array}$ & \begin{tabular}{|l|}
$\begin{array}{l}\text { Facultad de } \\
\text { ciencias }\end{array}$ \\
\end{tabular} & $\begin{array}{l}\text { Facultad de } \\
\text { ciencias de la } \\
\text { salud }\end{array}$ & $\begin{array}{l}\text { Facultad de } \\
\text { derecho }\end{array}$ & \begin{tabular}{|l|} 
Facultad de \\
educación
\end{tabular} & $\begin{array}{l}\text { Facultad de } \\
\text { filosofía y } \\
\text { letras }\end{array}$ & $\begin{array}{l}\text { Escuela } \\
\text { politécnica } \\
\text { superior }\end{array}$ \\
\hline Erasmus + & $\checkmark$ & $\checkmark$ & $\checkmark$ & $\checkmark$ & $\checkmark$ & $\checkmark$ & $\checkmark$ \\
\hline PPI & $\checkmark$ & & & & & $\checkmark$ & \\
\hline $\begin{array}{l}\text { Movilidad } \\
\text { global }\end{array}$ & $\checkmark$ & $\checkmark$ & $\checkmark$ & $\checkmark$ & $\checkmark$ & $\checkmark$ & $\checkmark$ \\
\hline AITANA & & & $\checkmark$ & & $\checkmark$ & & \\
\hline SICUE & $\checkmark$ & $\checkmark$ & $\checkmark$ & $\checkmark$ & $\checkmark$ & $\checkmark$ & $\checkmark$ \\
\hline DRAC & & $\checkmark$ & & & $\checkmark$ & $\checkmark$ & \\
\hline $\begin{array}{l}\text { Movilidad } \\
\text { fuera de } \\
\text { programas }\end{array}$ & $\checkmark$ & & $\checkmark$ & $\checkmark$ & & $\checkmark$ & \\
\hline $\begin{array}{l}\text { Beca } \\
\text { iberoamericana } \\
\text { Santander }\end{array}$ & $\checkmark$ & & $\checkmark$ & & $\checkmark$ & $\checkmark$ & \\
\hline $\begin{array}{l}\text { Leonardo Da } \\
\text { Vinci }\end{array}$ & & $\checkmark$ & & & & & \\
\hline IA Este & & $\checkmark$ & & & & & \\
\hline $\begin{array}{l}\text { Cámara de } \\
\text { comercio }\end{array}$ & & & $\checkmark$ & & $\checkmark$ & & \\
\hline $\begin{array}{l}\text { Otros } \\
\text { programas }\end{array}$ & & & $\checkmark$ & $\checkmark$ & $\checkmark$ & & \\
\hline
\end{tabular}

Como podemos ver en esta primera tabla, las facultades que más promueven la movilidad de su alumnado son la Facultad de Ciencias de la Salud y la Facultad de Educación a través de 8 becas ofertadas para su alumnado. Por otro lado, la facultad que menos la promueve es la Escuela Politécnica Superior con solo 3 programas de movilidad. En el punto intermedio el resto de centros con aproximadamente 6 propuestas de movilidad cada una. 
Con respecto a la tipología de becas, la mayoría son ayudas de estudios y solo las becas AITANA (ERASMUS+ prácticas) son de prácticas o contienen períodos de prácticas. Además, también encontramos movilidad para prácticas dentro del apartado otros programas para la Facultad de Ciencias de la Salud. Aquí, las Cámaras de Comercio de España y otras instituciones o empresas, ofrecen períodos de prácticas como las becas Faro global, becas Argo global, Eurodisea, programa Vulcanus en Japón, programa de la Leibniz University Hannover, prácticas en instituciones de la UE, etc. Asimismo, la facultad también ofrece la posibilidad de realizar prácticas extracurriculares en el extranjero. En general, con respecto a las prácticas, esto es lo mismo que se ofrece para el alumnado de la Facultad de Educación. En la Facultad de Ciencias encontramos las becas Leonardo Da Vinci y la IAEste que son programas de estancias remuneradas en países extranjeros.

También hay becas de movilidad, en el apartado otros programas, para el alumnado de la Facultad de Derecho. En este caso, no son programas de prácticas sino un Programa Propio de la UA llamado UAUNIVALI que promueve el intercambio entre alumnado de la UA y de Brasil. También el programa DRAC persigue el intercambio de estudiantes entre universidades, en este caso, dentro de la Xarxa Vives y para todo el colectivo de la universidad incluyendo PDI y PAS. Así, se trata de una ayuda para asistir a cursos, seminarios o congresos, para visitar un servicio de otra universidad, iniciar una investigación o un proyecto, cursar asignaturas u organizar actividades culturales.

Dentro de los programas de movilidad encontramos uno con origen en una institución privada. Las becas Iberoamericana Santander Grado que, como su nombre indica, promueven el intercambio de estudiantes de grado entre universidades de América del Norte (México y Puerto Rico) o Sudamérica (Argentina, Brasil, Colombia, Chile, Perú y Uruguay) y España o Portugal son financiadas por el Banco Santander. Con el fin de crear vínculos y favorecer el desarrollo de las instituciones, el alumnado puede reconocer los créditos cursados durante el semestre de intercambio, gracias a un convenio entre ambas universidades.

Aunque no es motivo de nuestro análisis, mención aparte merecen la beca PPI (Programa Propio para el Fomento de las Relaciones Internacionales) que es un programa para la movilidad del profesorado (PDI); y la beca ERASMUS + disponible, también, para el Personal de Administración y Servicios de la UA. Pero la beca ERASMUS+ nos interesa desde el punto de vista del alumnado universitario y como una de las becas más promovidas dentro de la Universidad de Alicante, ya que se oferta en todos sus centros y para todo su alumnado. Al igual que ocurre con la beca de movilidad global y el Sistema de Intercambio entre Centros Universitarios de España (SICUE).

Por las características de estas últimas (ERASMUS+, movilidad global y SICUE) y las de las becas AITANA (pertenece al programa ERASMUS+) y movilidad fuera de programas (se da en 4 facultades y 
es propio de la UA), hemos seleccionado estas para realizar un análisis detallado del contenido de sus bases de convocatoria.

\title{
3.2 CONOCER LAS CARACTERÍSTICAS DE LOS PROGRAMAS DE MOVILIDAD QUE OFRECE
}

\author{
LA UA.
}

\$abla 2. Requisitos de movilidad por programas./

\begin{tabular}{|c|c|c|c|c|c|c|c|}
\hline $\begin{array}{l}\text { Becas de } \\
\text { movilidad }\end{array}$ & Lugar & Propósito & $\begin{array}{l}\text { Requisitos } \\
\text { lingüísticos }\end{array}$ & $\begin{array}{l}\text { Requisitos } \\
\text { académicos }\end{array}$ & Requisitos administrativos & Duración & Cuantía \\
\hline Erasmus+ & $\begin{array}{l}\text { UE (KA103) } \\
\text { Argelia, } \\
\text { Colombia, } \\
\text { Nueva } \\
\text { Zelanda y } \\
\text { Túnez } \\
\text { (KA107) }\end{array}$ & Estudios & $\begin{array}{l}\text { General } \\
\text { B1(MCER) }\end{array}$ & $\begin{array}{l}\text { Cursado } \\
\text { min. } 60 \\
\text { créditos } \\
\text { (BOUA } 5 \text { de } \\
\text { noviembre } \\
\text { de 2010) }\end{array}$ & $\begin{array}{l}\text { Antes: Matrícula en UA, } \\
\text { Formulario de solicitud, } \\
\text { Acuerdo de aprendizaje, } \\
\text { Alojamiento, viaje y visado, } \\
\text { Seguro médico } \\
\text { Durante: matriculación destino, } \\
\text { confirmación estancia y } \\
\text { acuerdo académico definitivo } \\
\text { Final: certificado académico, } \\
\text { informe del estudiantes y } \\
\text { reconocimiento de créditos }\end{array}$ & $\begin{array}{l}\text { Semestral } \\
\text { (5meses) o } \\
\text { anual (10 } \\
\text { meses) } \\
\text { según } \\
\text { universidad } \\
\text { de destino }\end{array}$ & $\begin{array}{l}\text { Determinada } \\
\text { según } 3 \\
\text { categorias }\end{array}$ \\
\hline $\begin{array}{l}\text { Movilidad } \\
\text { Global }\end{array}$ & $\begin{array}{l}\text { Fuera de UE } \\
34 \text { países }\end{array}$ & Estudios & $\begin{array}{l}\text { Inglés B2 } \\
\text { Francés B2 } \\
\text { Árabe A2 } \\
\text { (MCER) } \\
\text { Universidades } \\
\text { de habla } \\
\text { hispana no hay } \\
\text { requisito }\end{array}$ & $\begin{array}{l}\text { Cursado } \\
\text { min. } 60 \\
\text { créditos } \\
(\text { BOUA } 5 \text { de } \\
\text { noviembre } \\
\text { de 2010) }\end{array}$ & $\begin{array}{l}\text { Antes: Matrícula en UA, } \\
\text { Formulario de solicitud, } \\
\text { Acuerdo de aprendizaje, } \\
\text { Alojamiento, viaje y visado, } \\
\text { Seguro médico } \\
\text { Durante: matriculación destino, } \\
\text { confirmación estancia y } \\
\text { acuerdo académico definitivo } \\
\text { Final: certificado académico, } \\
\text { informe del estudiantes y } \\
\text { reconocimiento de créditos }\end{array}$ & Semestre & $\begin{array}{l}\text { Entre } 450 € \text { y } \\
650 € \text { según el } \\
\text { grupo. También } \\
\text { ayuda final de } \\
250 € \text { y su es } \\
\text { estudiante con } \\
\text { beca MECD } \\
200 € \text { mensuales. }\end{array}$ \\
\hline
\end{tabular}

El primero, ERASMUS+ estudios, es un programa financiado por la UE (Erasmus Charter for Higher Education, ECHE) para la movilidad de alumnado que quiere realizar parte de sus estudios en otras universidades europeas. Las universidades adscritas a este programa realizan el reconocimiento de los créditos ECTS realizados en otras universidades. En este programa existen dos modalidades, en la KA103 la movilidad se realiza entre países europeos y en la KA107 entre países asociados que no participan en el programa. A modo de ejemplo, en la convocatoria 2019-2020 los destinos son Argelia, Colombia, Nueva Zelanda y Túnez. Las personas solicitantes del programa tienen dos periodos para formalizarla a través de la plataforma virtual de la institución (UACLOUD). Los requisitos que deben certificar (véase tabla 2) corresponden a la normativa de movilidad de la UA. La cuantía de la beca se determina en función del destino, una ayuda mensual que se establece en función de tres categorías y según la renta del país de acogida: el grupo 1, tiene $400 €$ de ayuda, el grupo 2, unos $350 €$ y el grupo 3, unos $300 €$. La duración de la estancia puede ser de un semestre o un curso académico según determine la universidad receptora. 
La beca de Movilidad global, también llamada no europea, es un programa propio de la UA que permite la movilidad del alumnado universitario fuera de Europa; a través de convenios y mediante el reconocimiento de los créditos cursados en otras universidades. Esta ayuda está subvencionada por el Vicerrectorado de Relaciones Internacionales y las distintas facultades o centros. La solicitud se puede realizar en dos convocatorias y el intercambio incluye instituciones, que tengan acuerdo de intercambio con la UA, en países como Argelia, Argentina, Australia, Azerbayán, Bolivia, Brasil, Canadá, Chile, China (Republica Popular), Colombia, Corea del Sur, Costa Rica, Cuba, E.E.U.U., Ecuador, Georgia, Hong Kong, India, Irán, Japón, Kazjistán, Malasia, México, Nueva Zelanda, Perú, Puerto Rico, Republica Serbia, Rusia, Suiza, Taiwán, Tailandia, Túnez, Uruguay y Vietnam. Los requisitos lingüísticos dependen del país de destino y la duración de la misma es de un semestre. La cuantía también está determinada por el país de destino y es variable (véase tabla 2). Además, podemos mencionar que esta ayuda es compatible con la beca Iberoamericana Santander Grado; por tanto, el alumnado de las Facultades de Económicas y Empresariales, Ciencias de la Salud, Educación y Filosofía y Letras (véase tabla 1) puede solicitar las dos si su destino coincide en ambas convocatorias (pto3.5 bases convocatoria curso 2019-2020 Programa de Movilidad Global).

Tabla 2. Requisitos de movilidad por programas.

\begin{tabular}{|c|c|c|c|c|c|c|c|}
\hline $\begin{array}{l}\text { Becas de } \\
\text { movilidad }\end{array}$ & Lugar & Propósito & $\begin{array}{l}\text { Requisitos } \\
\text { lingüísticos }\end{array}$ & $\begin{array}{l}\text { Requisitos } \\
\text { académicos }\end{array}$ & Requisitos administrativos & Duración & Cuantía \\
\hline AITANA & UE & Prácticas & $\begin{array}{l}\text { Entrevista para } \\
\text { cubrir nivel } \\
\text { solicitado por } \\
\text { empresa y } \\
\text { prueba de nivel } \\
\text { en la } \\
\text { plataforma } \\
\text { OLS de la } \\
\text { Comisión } \\
\text { Europea. }\end{array}$ & $\begin{array}{l}\text { Grado haber } \\
\text { cursado } \\
50 \% \text { plan de } \\
\text { estudios }\end{array}$ & $\begin{array}{l}\text { Antes: Solicitud inscripción, } \\
\text { proceso de preselección y } \\
\text { envío de currículums a } \\
\text { empresas. } \\
\begin{array}{l}\text { Durante: confirmación de } \\
\text { llegada, informe intermedio }\end{array} \\
\text { Final: memoria final de } \\
\text { prácticas, informe final } \\
\text { AITANA- Erasmus + }\end{array}$ & $\begin{array}{l}\text { Máx. } 12 \\
\text { meses entre } \\
\text { todos los } \\
\text { Erasmus. } \\
\text { Sin } \\
\text { suplementos } \\
3 \text { meses }\end{array}$ & $\begin{array}{l}\text { Determinada } \\
\text { según } 3 \\
\text { categorías } \\
\text { Complemento } \\
\text { ayuda de UA } \\
\text { máx. } 650 € \text { mes }\end{array}$ \\
\hline SICUE & $\begin{array}{l}\text { Universidades } \\
\text { españolas }\end{array}$ & Estudios & & $\begin{array}{l}\text { Tener } \\
\text { superados } \\
45 \text { ECTS y } \\
\text { estar } \\
\text { matriculado } \\
\text { de al menos } \\
\text { 30 ECTS. }\end{array}$ & $\begin{array}{l}\text { Antes: Matrícula en UA, } \\
\text { Formulario de solicitud, } \\
\text { Acuerdo de aprendizaje, } \\
\text { Alojamiento y viaje } \\
\begin{array}{l}\text { Durante: matriculación destino, } \\
\text { confirmación estancia y } \\
\text { acuerdo académico definitivo }\end{array} \\
\text { Final: certificado académico y } \\
\text { reconocimiento de créditos }\end{array}$ & $\begin{array}{l}\text { Un semestre } \\
\text { o un curso } \\
\text { académico. }\end{array}$ & \\
\hline $\begin{array}{l}\text { Movilidad } \\
\text { fuera de } \\
\text { programas }\end{array}$ & $\begin{array}{l}\text { Universidades } \\
\text { extranjeras } \\
\text { distinta de los } \\
\text { otros } \\
\text { programas }\end{array}$ & Estudios & $\begin{array}{l}\text { B1 idioma país } \\
\text { destino. }\end{array}$ & $\begin{array}{l}\text { Cursado } \\
\text { mín. } 60 \\
\text { créditos. }\end{array}$ & $\begin{array}{l}\text { Carta de aceptación de la } \\
\text { universidad de destino }\end{array}$ & & $\begin{array}{l}\text { No hay ayuda } \\
\text { económica. }\end{array}$ \\
\hline
\end{tabular}


La beca AITANA es un programa que incluye a todos los miembros de las instituciones $u$ organizaciones que se encargan de la educación y formación profesional.

Con respecto a los estudiantes, la Universidad de Alicante solicita fondos Erasmus (Erasmus Charter for Higher Education, ECHE) de la UE para cofinanciar prácticas en entidades colaboradoras ubicadas en países que participen en el programa de movilidad. Los destinos previstos pueden ser empresas, asociaciones, centros educativos de cualquier nivel de enseñanza, centros de investigación, ONGs o cualquier entidad pública o privada ubicada en países miembros de la UE (más Islandia, Liechtenstein, Noruega, Turquía y Macedonia). La duración máxima de las prácticas dependerá del resto de las movilidades Erasmus que se hayan realizado tanto dentro del nuevo programa Erasmus + como en el anterior Erasmus. Cada estudiante puede realizar, dentro de cada ciclo de estudios superiores, movilidades con una duración máxima de 12 meses. Por tanto, las becas ERASMUS son compatibles entre sí y se pueden suplementar con otras ayudas de la UA o la empresa de acogida. Como hemos determinado hasta ahora, la cuantía de la ayuda está en función del país de destino (véase ERASMUS+ estudios). Aunque esta depende de la pertinencia a un grupo u otro (tres grupos). Durante los 3 meses con beca AITANA ( $\sin$ suplementos; véase tabla 2), la UA aportará una cantidad complementaria hasta llegar a $\operatorname{los} 650 € / m e s$. Además, con la financiación exclusiva de la UA (650 €/mes), las prácticas podrán prolongarse al menos 1 mes más, o más de 4 meses con la financiación que aporte la empresa de acogida o la ayuda ERASMUS+ y la UA. En estos casos, debe tenerse en cuenta que el período de prácticas no puede ir más allá del 30 de septiembre y/o sobrepasar los 12 meses de ERASMUS (total becas del programa). Por otro lado, los solicitantes deberán cumplir los requisitos linguísticos, académicos y administrativos determinados por todas las instituciones (véase tabla 2).

Las becas de la movilidad del Sistema de Intercambio entre Centros Universitarios Españoles (SICUE) tiene convocatoria única y sirven para el alumnado que desee realizar una parte de sus estudios en otra universidad española. El objetivo de este programa es garantizar el reconocimiento académico de los estudios cursados en la institución de destino (ECTS) y el aprovechamiento personal y cultural que tiene realizar estudios en otros contextos manteniendo el mismo perfil curricular y la matrícula en la Universidad de Alicante. La solicitud de este programa requiere una serie de requisitos académicos y administrativos (véase tabla 2) que los participantes deben cumplir. Asimismo, en esta beca podrá participar alumnado comunitario y no comunitario, residente en España, y beneficiarios de otros programas de intercambio.

La movilidad fuera de programas que reconoce la UA está regulada conforme a la normativa interna del BOUA del 5 de noviembre de 2010 normativa de movilidad de estudiantes de la Universidad de Alicante. En esta, el alumnado podrá proponer, al Vicedecanato de Relaciones Internacionales de la 
facultad, la realización de una estancia en una universidad extranjera distinta de los programas de movilidad convocados por la Universidad de Alicante. Además de lo requerido por la normativa, el alumnado que participe en este programa deberá cumplir los requisitos indicados (véase tabla 2). A los beneficiarios se les reconocerán los estudios cursados, aplicando la Normativa de Movilidad de Estudiantes de la Universidad de Alicante, mediante un acuerdo académico o contrato de estudios donde se especificará las asignaturas que se cursarán en la universidad de destino y su equivalencia en la Universidad de Alicante. Así, la duración del programa dependerá de este acuerdo y las posibilidades del alumnado. No obstante, al tratarse de una iniciativa personal, no tendrán derecho a obtener subvenciones o ayudas económicas para cubrir sus gastos de matrícula o estancia.

\subsection{ANALIZAR LAS DIFERENCIAS QUE PRESENTAN LOS DISTINTOS CENTROS DE LA UA} CON RESPECTO A LA POSIBILIDAD QUE TIENE SU ALUMNADO DE ACCESO A BECAS DE MOVILIDAD.

A modo de resumen señalaremos que las facultades que más se benefician de estos programas son las de Ciencias de la Salud y Educación, que cuentan con un mayor número de programas ofertados, y la de Ciencias que dispone de dos becas específicas para su campo. Asimismo, la posibilidad de acogerse a un programa de movilidad para realizar prácticas remuneradas parece limitarse a las facultades citadas anteriormente y a la Facultad de Derecho, excluyendo al resto de centros o limitando estas prácticas a movilidades fuera de programas con contactos que puedan encontrar por su cuenta y que no tienen ayuda económica. Además, el alumnado de la Escuela Politécnica Superior ni siquiera cuenta con esta opción. Estos son, sin lugar a dudas, los más perjudicados por las políticas de movilidad de la UA con solo tres programas a su disposición.

Con respecto a las cuantías de las becas, las cantidades de la mayoría de programas requieren que las familias tengan dinero para poder complementar los gastos que suponen mandar a un estudiante fuera de casa y que, en la mayoría de los casos, no se cubren con las ayudas. Aunque la Universidad de Alicante intenta compensarlo con aportaciones propias, estos importes no suelen ser suficientes, por tanto, reconocemos un buen número de alumnas y alumnos que no podrán participar de estos programas de movilidad por falta de recursos. Lo que establece una discriminación por cuestiones económicas en el acceso a la movilidad universitaria y, consecuentemente, a las posibilidades de empleabilidad en según que carreras universitarias. No olvidemos que muchos perfiles laborales ya exigen idiomas, estancias en el extranjero y prácticas en empresas reconocidas internacionalmente.

Los requisitos de acceso a los programas de movilidad son otra de las cuestiones a destacar. En lo que respecta al requisito lingüístico, normalmente, no se solicita un nivel de idiomas muy elevado, 
situándose entre el A2 y el B2 del Marco Común Europeo de Referencia (MCER) y siendo el B1 el más común, por lo que parece una petición realista. No hemos de olvidar que actualmente se solicita un nivel B1 de una lengua extranjera para finalizar los estudios de Grado; y que resulta casi impensable poder cursar una asignatura, en una lengua extranjera, sin un nivel superior. Aunque, como ya hemos visto, la preparación en lenguas extranjeras de nuestro sistema aún está lejos del nivel óptimo para la movilidad (Eurydice, 2017). En cuanto a los requisitos académicos (min. 60 ECTS), estos implican la posibilidad de solicitar la movilidad a partir del segundo curso de Grado o en el primero para la movilidad dentro de España. Con respecto al programa AITANA (ERASMUS+ prácticas) se requiere haber cursado la mitad del plan de estudios. En todos los casos parece bastante razonable acogerse a algún programa de movilidad o prácticas laborales cuando ya se tienen unos conocimientos mínimos sobre el sistema universitario, el perfil curricular y las características del puesto a desempeñar. Otra cuestión son los requisitos administrativos que tienen las becas, desde la solicitud de movilidad hasta la vuelta a la universidad y la solicitud de reconocimiento de créditos. Algunos de ellos nos parecen excesivos y en muchos casos innecesarios. La burocracia para el alumno tiende a ser redundante, duplicidad de documentos, y se repite mucha información por no disponer de un sistema de comunicación e intercambio de documentos eficaz entre la universidad de origen y de destino. Incluso, problemas para el intercambio telemático de documentos, que facilite el proceso, elimine el trasiego de papeles y organice la información que el alumnado participante debe entregar a la universidad de origen para justificar la estancia y los gastos.

Finalmente hemos de indicar que en el total de programas de movilidad para el alumnado de la UA, hemos recogido 5 de prácticas y 6 de estudios, se realiza un reparto bastante equitativo del propósito de las becas. Por lo que respecta a la dispersión geográfica, las cinco becas más destacadas reconocen la movilidad en España (SICUE), dentro de la Unión Europea (Erasmus+) y fuera de ella (Movilidad global). Posibilitando, además, la movilidad en universidades fuera de los programas estipulados. Con respecto a la duración de la movilidad, era de esperar que esta se ajustara al calendario académico de las universidades, ya que esto facilita el seguimiento de las asignaturas y la evaluación de las mismas. Aunque se debe tener en cuenta que en algunos países, como los ubicados en América del Sur, el calendario académico no transcurre de septiembre a junio como en España por lo que esta cuestión se debe flexibilizar.

\section{DISCUSIÓN Y CONCLUSIONES FINALES}

Como hemos podido ver a raíz de los datos recogidos y las tablas elaboradas, la Universidad de Alicante se acoge a muchos de los planes de movilidad ofertados dentro de la Unión Europea para facilitar el acceso de su alumnado a una cultura global que mejore sus competencias y, consecuentemente, su 
empleabilidad (Cairns, Krzaklewska, Cuzzocrea, \& Allaste, 2018). En toda la UE se procura adecuar las asignaturas cursadas en las universidades a los perfiles académicos y se procura garantizar, con más o menos éxito, el reconocimiento de los créditos (ECTS) cursados por el alumnado (Eurydice, 2017). Ambas políticas son fundamentales para trabajar a favor de los principios de calidad, movilidad, diversidad y competitividad universitarios (EEES, 2019; MECD, 2019).

Pero estas prácticas no son suficientes y los datos así lo corroboran (Eurydice, 2017; Unidad Técnica de Calidad de la Universidad de Alicante, 2019) cuando se habla de cifras de movilidad, información y orientación al alumnado, formación adecuada en lenguas extranjeras, portabilidad de becas, apoyo proporcionado a los estudiantes procedentes de un entorno socioeconómico desfavorecido para iniciar programas de movilidad (Sin, Tavares \& Neave, 2017) y reconocimiento de los ECTS y las titulaciones (Eurydice, 2017). Aunque inicialmente esta pueda ser una experiencia muy atractiva para el alumnado universitario (Donat, Carmona, Vidal, \& Benlloch, 2018; Sin, Tavares \& Neave, 2017) por las posibilidades de crecimiento personal, cultural y laboral que tiene la experiencia (Aguirre, Cruz y Banda, 2018; Cairns, Krzaklewska, Cuzzocrea, \& Allaste, 2018), hemos visto que el interés suele reducirse sustancialmente cuando llega el momento de solicitar las ayudas de los diversos programas de movilidad.

Por lo que, como en todo proceso, las políticas europeas y de la UA requieren de revisiones, nuevos enfoques y mejoras. Por ejemplo en las cuantías de las becas, dado que son también un activo determinante a la hora de seleccionar el programa (Nicholls, 2018) y un factor discriminante para acceder a la movilidad (Cuzzocrea \& Cairns, 2020). Así, resulta necesario adecuar las cantidades para favorecer que todo el alumnado, y no solo las familias con más recursos, puedan acceder a estas. De acuerdo con los objetivos de Europa para 2020, la movilidad debe ser una posibilidad para todo el alumnado y nunca una razón para la desigualdad y la discriminación (Consejo Europeo, 2016; Yuval \& Miri, 2017) como ocurre actualmente en casi todos los contextos universitarios (European Commission/EACEA/Eurydice, 2020) y de los cuales la Universidad de Alicante no es una excepción.

También en temas de promoción y visibilización de los programas de movilidad para mejorar el acceso del alumnado a esta información (European Commission/EACEA/Eurydice, 2020). Por ejemplo en el posicionamiento de la información, poniendo en contacto alumnado que se interesa por programas de movilidad y otros que participan de ellos, creando recursos web y en RRSS para el intercambio de experiencias, etc. Asimismo, es imprescindible que el alumnado que participa en los programas de movilidad obtenga el apoyo de las instituciones de origen y acogida para superar el miedo que supone dar el paso y, así, favorecer su adaptación al cambio cultural y social que se produce durante la movilidad. Con este objetivo, se debería crear una buena red de contactos para apoyar al alumnado que recibimos y 
al que enviamos. Esto permite contactos interuniversitarios que favorecen la movilidad de toda la Comunidad Educativa (Ramírez, \& Ortega, 2019).

Por otro lado, desde la institución se debería trabajar para optimizar la burocracia, favoreciendo el contacto institucional y permitiendo el intercambio telemático de la información, lo que facilitaría los trámites académicos y administrativos a las y los futuros participantes de estos programas dado que, como ya se comentaba antes, los procedimientos de la actual normativa resultan redundantes y los contactos insuficientes para llevar a cabo programas de prácticas en todas las facultades.

Es por esto que para futuras investigaciones resultaría interesante esclarecer por qué las ingenierías solo cuentan con la posibilidad de realizar 3 programas de movilidad y no tienen programas de prácticas como otras facultades; también por qué no se favorece la movilidad fuera de programas de este alumnado y del de ciencias. Y, complementariamente, qué ocurre con los programas de prácticas en las facultades de Derecho, Económicas y Empresariales y Filosofía y Letras. Con esto, podríamos dar una visión más completa de la situación incluyendo información privilegiada del Secretariado de Movilidad y/o el Vicerrectorado de Relaciones Internacionales.

Asimismo, otra línea de investigación interesante sería indagar, a través de entrevistas con un enfoque cualitativo, en las razones por las que el alumnado no se plantea realizar programas de movilidad durante su formación inicial: nivel de conocimiento de los programas, manejo del idioma, primeras separaciones del núcleo familiar, etc. Estos datos pueden ayudar para tratar de mejorar los porcentajes de participación en estos programas y limitar la exclusión del alumnado como ya lo han hecho las investigaciones sobre: las razones para no iniciar la movilidad tras conocer los requisitos de estos programas o los informes y las entrevistas realizadas al alumnado recibido en la movilidad (ERASMUS+, AITANA y Movilidad Global).

Finalmente, queremos destacar la importancia de investigar en atención a la diversidad e inclusión como medio para conocer la adecuación, de las políticas implementadas en las instituciones, a todos los miembros de la sociedad analizando los procesos y la opinión de sus participantes. En el ámbito educativo, esto permite asegurar el acceso, la calidad de la educación y la no discriminación de parte de la Comunidad Educativa. Como miembros activos de la Comunidad Universitaria, el alumnado, en continua evolución, favorece la transformación de la sociedad hacia una conciencia ética, más igualitaria y participativa. Así pues, privarles de la igualdad de oportunidades en cualquier ámbito es condenar a la sociedad a la precariedad y la mediocridad. Además, no tenerles en cuenta es adoptar una posición dominante que en nada favorece la democratización del sistema educativo. En este sentido, son necesarias políticas que aseguren la inclusión del alumnado en todos los procesos de la educación. 


\section{REFERENCIAS}

Aguirre, F., Cruz, M. A., y Banda, M. I. (2018). La dimensión sociocultural de la globalización en relatos sobre una experiencia de movilidad internacional universitaria. Revista Latinoamericana de Ciencias Sociales, Niñez y Juventud, 16(2), 881-895. doi: 10.11600/1692715x.16216

Arroyo, S., \& Lozano, I. (2020). El alumnado ERASMUS en el Prácticum a maestro/a: estudio de caso. En: Roig-Vila, Rosabel (ed.). La docencia en la Enseñanza Superior. Nuevas aportaciones desde la investigación e innovación educativas (pp. 1023-1033): Barcelona: Octaedro.

BET. (2011). Informe del proyecto de expertos de Bolonia 2011-2013. OAPEE: MECD. Recuperado de http://sepie.es/doc/informe-bet-2011-2013---revisado.pdf

Consejo Europeo. (2016). Cooperación de la Unión Europea en el ámbito de la educación y la formación. Recuperado de https://eur-lex.europa.eu/legal-content/ES/TXT/?uri=LEGISSUM\%3Aef0016

Cairns, D., Krzaklewska, E., Cuzzocrea, V., \& Allaste, A. A. (2018). Mobility, education and employability in the European Union. Inside Erasmus. Springer International Publishing AG. doi:10.1007/978-3-319-76926-4

Cuzzocrea, V., \& Cairns, D. C. (2020). Mobile moratorium? The case of young people undertaking international internships. Mobilities. doi: 10.1080/17450101.2020.1724611

Donat, M. A., Carmona, C., Vidal, J., \& Benlloch, M. J. (2018). Competencias interculturales en Educación Superior: Aspecto clave para la movilidad. Education in the Knowledge Society (EKS), 19(1), 97- 114. doi: https://doi.org/10.14201/eks201819197114

EEES. (2019). Desarrollo cronológico. Recuperado de http://www.eees.es/es/eees-desarrollo-cronologico

European Commission/EACEA/Eurydice. (2020). The European Higher Education Area in 2020: Bologna Process Implementation Report. Luxembourg: Publications Office of the European Union. doi:10.2797/756192

Eurydice. (2017). Boletín informativo de Eurydice $n^{\circ}$ 2. Panel de Indicadores de la Movilidad. Informe sobre la situación de la Educación Superior - Edición 2017. Secretaría General Técnica. Recuperado de https://sede.educacion.gob.es/publiventa/boletin-informativo-de-eurydice-n-2-panel-de-indicadores-dela-movilidad-informe-sobre-la-situacion-de-la-educacion-superior-edicion-2017/educacion-superiormovilidad/22125

MECD. (2019). Gestión de títulos universitarios. Recuperado de http://www.educacionyfp.gob.es/educacion-mecd/areas-educacion/estudiantes/ensenanzasuniversitarias/gestion-titulos/suplemento-europeo.html

Nicholls, S. (2018). Influences on International Student Choice of Study Destination: Evidence from the United States. Journal of International Students, 8(2), 597-622. doi: 10.5281/zenodo. 1249043

Ramírez, A., \& Ortega, J. C. (2019). El impacto institucional de la movilidad internacional estudiantil. El caso de la Universidad Veracruzana. Revista de Investigación Educativa, 28(2), 207- 233. doi: https://doi.org/10.25009/cpue.v0i28.2605 
Ramos, L. R., Sánchez, S., Saucedo, \& Cortés, A. (2021). Student Mobility Program in BINE's Bachelor's Degree in Special Education. South Florida Journal of Development, Miami, 2(1), 643-650. doi: https://doi.org/10.46932/sfjdv2n1-047

Sin, C., Tavares, O., \& Neave, G. (2017). Student Mobility in Portugal: Grappling With Adversity. Journal of Studies in International Education, 21(2), 120-135. doi: 10.1177/1028315316669814

Unidad Técnica de Calidad de la Universidad de Alicante. (2019). La UA en cifras 2018: anuario estadístico de la Universidad de Alicante. Recuperado de https://utc.ua.es/es/documentos/ua-encifras/libros-ua-en-cifras/ua-en-cifras-2018.pdf

Yuval D. \& Miri Y. (2017). Mobility as a continuum: European commission mobility policies for schools and higher education. Journal of Education Policy, 32(2), 198-210. doi:10.1080/02680939.2016.1243259 